\title{
Simulation of Angular Acceleration Control for Information Acquisition Robots with Air-Ground Purpose
}

\author{
Chunping Liu*, Xiangbin Zhao, and Huimin Gao \\ Intelligent Manufacturing College, Tianjin Sino-German University of Applied Science, Tianjin, 300000, China
}

\begin{abstract}
In order to allow information acquisition robots to run steadily on land and in the air, it is necessary to study the angular acceleration control method for ground-air dual-purpose information acquisition robots. When the angular acceleration control method for the current robot is used to control the angular acceleration of ground-air dual-purpose information acquisition robots, there are problems of low control accuracy and low stability. Thus, a method of angular acceleration control for ground-air dual-purpose information acquisition robots is proposed. The dynamic equation of the information acquisition robot motion process is constructed according to the DC motor equation of the right and left track driving wheels and the driving motor, as well as the potential balance equation. According to the dynamic equation, the initial values of the information acquisition robot system and the inverse system are obtained. The state equation model of the information acquisition robot system is obtained by using the inverse method of the $\alpha$-order integral. On the basis of the state equation model, the angular acceleration regulator control model is obtained to control the angular acceleration of ground-air dual-purpose information acquisition robots. The simulation results show that the control results of the proposed method have high accuracy and stability.
\end{abstract}

Keywords: ground-air dual-purpose; information acquisition; robot; angular acceleration control

(Submitted on October 28, 2019; Revised on November 30, 2019; Accepted on December 15, 2019)

(C) 2019 Totem Publisher, Inc. All rights reserved.

\section{Introduction}

The angular acceleration of robots is controlled by various angular acceleration control and compensation techniques, and the angular acceleration control of ground-air dual-purpose information acquisition robots has been studied [1-3]. The current angular acceleration control method for information acquisition robot has no effect on the overall speed of robots. Considering the degree of control, there are problems of low control accuracy and low stability [4]. It is necessary to study the angular acceleration control method for ground-air dual-purpose information acquisition robots.

Zhao and Ma put forward a method for calibrating the angular acceleration of robot rotation. They connected the sensor and the excitation source coaxially, received the angular acceleration excitation source generated by the servo motor, and calculated the electrical parameters of the excitation source. According to the calculation results, the angular acceleration was obtained, and the voltage value of the angular acceleration sensor on the basis of the angular acceleration was measured to complete the angular acceleration control of information acquisition robots. However, there was an error between the measured acceleration and the actual acceleration, and the control accuracy of the method was low [5]. Peng et al. put forward an acceleration control method for information acquisition robots. They constructed a non-linear dynamic model of information acquisition robots based on the closed-loop control strategy of time-delay acceleration and Hamilton principle. The critical excitation amplitude and response peak value of the robot were computed by the multi-scale method. According to the calculation results, the angular acceleration of the information acquisition robot was controlled. This method could not control the output voltage in a short time to maintain stability, and the stability was low [6]. Geng et al. put forward an angular acceleration control method based on trajectory planning. By constructing an angular acceleration curve and calculating undetermined coefficients on the basis of residual vibration amplitude, the undetermined coefficients were

\footnotetext{
* Corresponding author.

E-mail address: liu123chun456ping@163.com
} 
brought into the angular acceleration curve to control the angular acceleration of information acquisition robots. This method considered fewer coefficients and had a lower control accuracy [7].

In summary, an angular acceleration control method for ground-air dual-purpose information acquisition robots is proposed in this paper.

\section{Overall Structure and Parameter Selection of Ground-Air Dual-Purpose Information Acquisition Robots}

\subsection{Overall Structure of Ground-Air Dual-Purpose Information Acquisition Robots}

The general structure of ground-air dual-purpose information acquisition robots is shown in Figure 1.

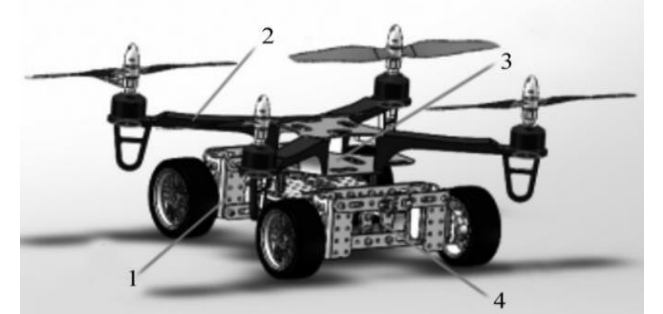

1. Walking mechanism; 2. Flying mechanism; 3. Computer control system; 4. Information acquisition system. Figure 1. Three-dimensional model of robot

It mainly consists of four parts: a ground walking mechanism, a flight mechanism, a control system, and an information acquisition system.

\subsection{Main Performance Indicators of Ground-Air Dual-Purpose Information Acquisition Robots for Agriculture}

In order to meet the performance requirements, the main performance indicators of the robots are shown in Table 1.

Table 1. Main performance parameters of robots

\begin{tabular}{cc}
\hline Parameter name & Parameter value \\
\hline Walking speed $/\left(\mathrm{m} \cdot \mathrm{min}^{-1}\right)$ & $7 \sim 10$ \\
Mass of whole machine $/ \mathrm{kg}$ & 5.5 \\
Volume size $/ \mathrm{mm}$ & $210 \times 87 \times 150$ \\
Required energy & Lithium battery \\
Flight speed $/\left(\mathrm{m} \cdot \mathrm{s}^{-1}\right)$ & $2 \sim 3$ \\
Flight altitude $/ \mathrm{m}$ & $0 \sim 20$ \\
\hline
\end{tabular}

\section{Design of Ground-Air Dual-Purpose Information Acquisition Robots for Agriculture}

\subsection{Design of Ground Walking Mechanism}

The ground walking mechanism of the machine is shown in Figure 2. The choice of an Al-Mg alloy as the structural material of the ground walking mechanism greatly reduces the weight of the structure and provides a guarantee for the subsequent realization of the aircraft's air flight [8-9]. When a robot works in the field, it usually has to overcome great ground resistance, so this machine adopts a four-wheel drive mode with four circumferential actuators to provide power and achieve the purpose of outputting large torque. In order to adapt to complex topography and landform, a four-bar mechanism with flexible swing is adopted in the design to reduce the damage to the mechanism caused by vibration when crossing the gully and the influence on the results of information acquisition.

\subsection{Flight Agency Design}

The flight mechanism of the machine is designed based on the principle of quadrotors. The flight mechanism diagram is shown in Figure 3. Motors 1 and 3 rotate clockwise, and motors 2 and 4 rotate clockwise. Therefore, when the robot balances the flight, both the gyro effect and the aerodynamic torque effect are offset. Since the counter torque applied to the fuselage by each rotor is opposite to the direction of rotation of the rotor, motors 2 and 4 rotate clockwise while motors 1 
and motor 3 rotate counterclockwise. This can offset the counter-torque of the rotor to the fuselage, so that the whole machine can be balanced.

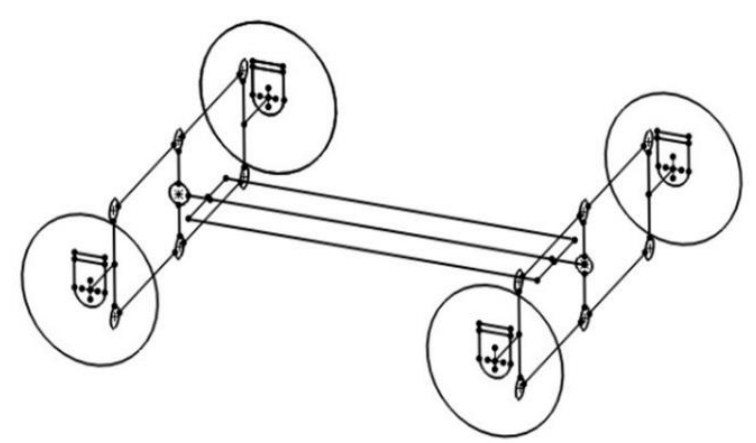

Figure 2. Schematic diagram of walking mechanism

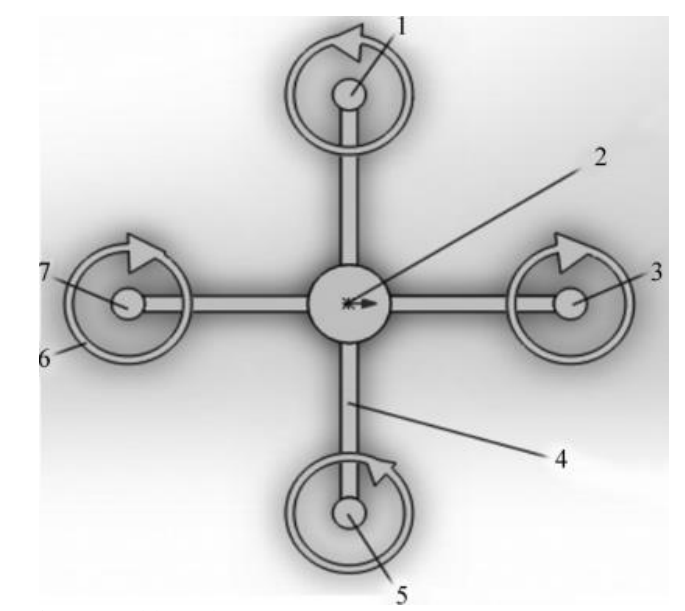

1. Motor 1; 2. Main control board; 3. Motor 4; 4. Support; 5. Motor 3; 6. Rotate; 7. Motor 2.

Figure 3. Flight structure diagram

\subsection{Control System Structure Design}

The structure of the robot control system is shown in Figure 4. The system uses the computer main control board with STM32F407 as the core. The main control board has eight PWM output ports, four of which are connected by an electronic governor and four brushless DC motors. The other four PWM output ports are connected to the four circumferential steering gears through the servo control module. The speed and steering of each motor are controlled by controlling the output level of the PWM output port. The functions of walking, flying, shifting, and posture adjustment of the robot are thereby realized. At the same time, the main control board is connected to the information collection module through the serial port, which can realize the control of the information collection module and the processing of the collected information.

\subsection{Principle of Angular Acceleration Control for Information Acquisition Robots}

When the angular acceleration control method for the current robot is used to control the angular acceleration of the ground-air dual-purpose information acquisition robot, the angular acceleration information of the relative inertial space of the robot is measured based on the rate helix. The Kalman filter and tracking differentiator are used to estimate and fuse the angular acceleration information of the information acquisition robot. The fusion result is used as the input of the disturbance information acquisition robot observer to control the angular acceleration of the information acquisition robot.

The motion equation and observation equation of information acquisition robots are as follows:

$$
\left\{\begin{array}{l}
x_{k+1}=\mathrm{A} x_{k}+w_{k} \\
z_{k}=H x_{k}+v_{k}
\end{array}\right.
$$




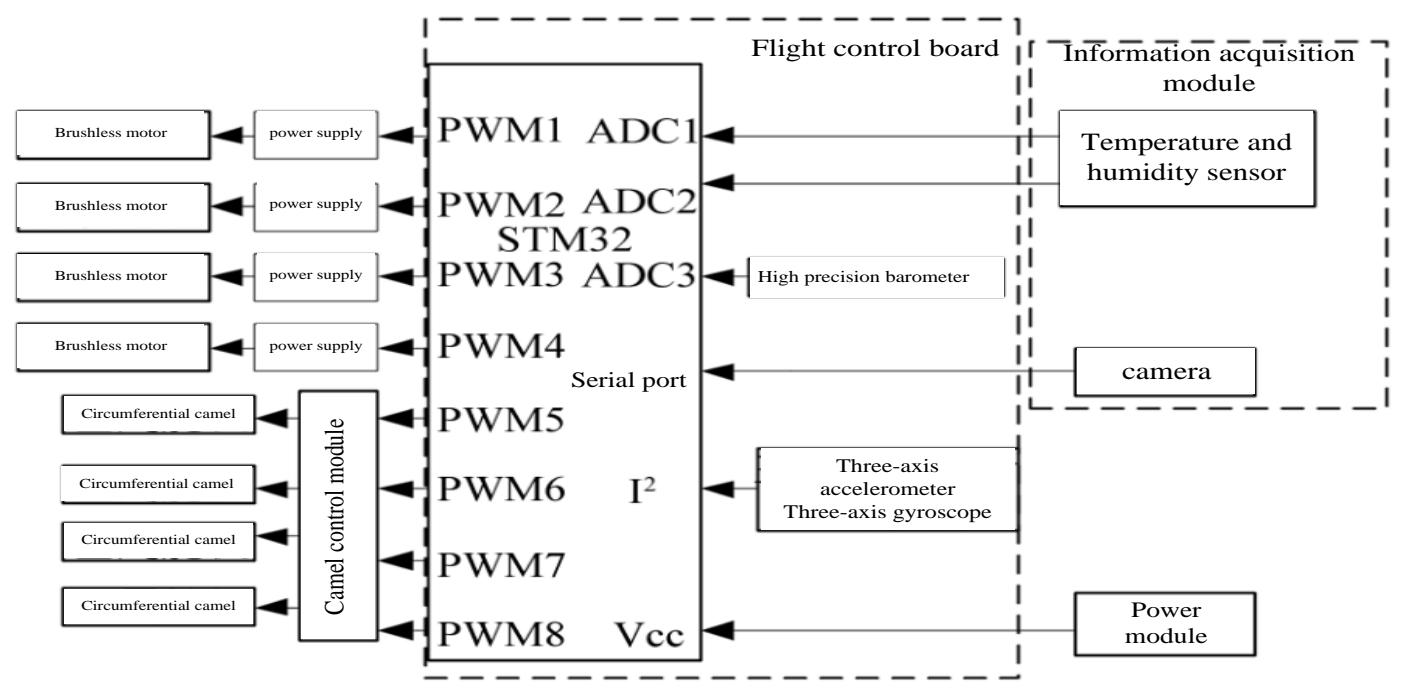

Figure 4. Control system block diagram

In Equation (1), $x_{k}$ denotes the state vector of the $k^{\text {th }}$ different period, $z_{k}$ denotes the dimension measurement value of the $k^{\text {th }}$ different period, $\mathrm{A}$ and $H$ denote the coefficient matrix, $w_{k}$ denotes the noise existing in the information acquisition robot system, and $v_{k}$ denotes the noise measured by the information acquisition robot.

The Kalman filter is expressed as

$$
\left\{\begin{array}{l}
\hat{x}_{k / k-1}=\mathrm{A} \hat{x}_{k-1} \\
\hat{x}_{k}=\widehat{x}_{k / k-1}+K_{k}\left(z_{k}-H \hat{x}_{k / k-1}\right) \\
K_{k}=P_{k / k-1} H^{T}\left(H P_{k / k-1} H^{T}+R\right)^{-1} \\
P_{k / k-1}=\mathrm{A} P_{k-1} \mathrm{~A}^{T}+Q \\
P_{k}=\left(I-K_{k} H\right) P_{k / k-1}
\end{array}\right.
$$

In Equation (2), $\hat{x}_{k / k-1}$ represents the predicted estimate of the state of the information acquisition robot, $\hat{x}_{k-1}$ represents the optimal estimate of the state of the information acquisition robot, $z_{k}$ represents the actual output, $P_{k / k-1}$ represents the prior error corresponding to $\hat{x}_{k / k-1}, P_{k}$ represents the posterior error corresponding to $\hat{x}_{k-1}, Q$ represents the variance matrix of the noise of the information acquisition robot, $R$ represents the variance matrix of the noise of measured noise, and $K_{k}$ represents the gain of the Kalman filter.

When the angular velocity of the robot is used to estimate the angular acceleration of the robot, set the following:

$$
x_{k}=\left[\begin{array}{l}
w_{k} \\
\beta_{k}
\end{array}\right], \quad A=\left[\begin{array}{ll}
1 & T \\
0 & 1
\end{array}\right], \quad H=\left[\begin{array}{ll}
1 & 0
\end{array}\right]
$$

In Equation (3), $w_{k}$ denotes the angular acceleration measured by the gyroscope of the information acquisition robot, $\beta_{k}$ denotes the estimated angular acceleration of the information acquisition robot, and $T$ denotes the sampling period of the information acquisition robot.

The tracking differentiator is a dynamic system, and the discrete tracking differentiator is expressed as

$$
\left\{\begin{array}{l}
\hat{x}_{1}(k+1)=\widehat{x}_{1}(k)+T \widehat{x}_{2}(k) \\
\hat{x}_{2}(k+1)=\widehat{x}_{2}(k)+T f s t\left(k, \widehat{x}_{2}(k), M, h\right)
\end{array}\right.
$$


In Equation (4), $(k)=\widehat{x}_{1}(k)-r(k)$ represents the error of input variable estimation of the information acquisition robot. $M$ represents the angular acceleration factor of the information acquisition robot, which affects the tracking speed, and $h$ represents the filtering factor.

The error between the angular acceleration control and the actual output of the information acquisition robot can be used to construct the evaluation index function [10-11]. According to the index function, the output signal of the speed control for the information acquisition robot is fused, which is expressed as

$$
J=\alpha \varepsilon(t)+\beta e^{-\lambda(t-x)}(\tau) \mathrm{d} \tau
$$

In Equation (5), $\alpha \geq 0, \beta>0$, and $\lambda>0 . \varepsilon(t)$ denotes the error between the actual output of the filter, and $\lambda$ denotes the forgetting factor, which can determine the memory length of the information acquisition robot. The angular acceleration parameters $\alpha$ and $\beta$ are selected reasonably, and the instantaneous error and historical error are adjusted. The angular acceleration of the information acquisition robot is controlled according to the results of the harmonization [12-13].

\section{Angular Acceleration Control Method for Ground-Air Dual-Purpose Information Acquisition Robots}

\subsection{Dynamic Equation of Ground-Air Dual-Purpose Information Acquisition Robots}

Through the DC motor equation and potential balance equation of ground-air dual-purpose information acquisition robots, the dynamic equation of the ground-air dual-purpose information acquisition robot is obtained, which provides information for acceleration control.

Multi-motor coordinated control is the motion control mode of ground-air dual-purpose information acquisition robots. The DC motor equation and potential balance equation are as follows:

$$
\begin{gathered}
J_{r}(t) \omega_{r}(t)+F \omega_{r}(t)+T_{d r}(t)=k_{t} i_{r}(t) \\
L i_{r}(t)+R i_{r}(t)+k_{e} \omega_{r}(t)=u_{r}(t) \\
J_{l}(t) \omega_{l}(t)+F \omega_{l}(t)+T_{d l}=k_{t} i_{l}(t) \\
L i_{l}(t)+R i_{l}(t)+k_{e} \omega_{l}(t)=u_{l}(t)
\end{gathered}
$$

Equations (6) and (8) are the moment balance equations of the driving motors on both sides of the information acquisition robot, and Equations (7) and (9) are the potential balance equation of the driving motors on both sides of the information acquisition robot. In the model, $L$ represents the armature inductance of the information acquisition robot, $R$ represents the armature resistance of the information acquisition robot, $J_{r}(t)$ represents the moment of inertia on the axis of the right drive wheel motor of the information acquisition robot, $J_{l}(t)$ represents the moment of inertia on the axis of the left drive wheel motor of the information acquisition robot, and $F$ represents the friction coefficient of the motor's output shaft of the information acquisition robot. $k_{e}$ represents the back EMF coefficient, $k_{t}$ represents the electromagnetic torque coefficient, $T_{d r}(t)$ and $i_{r}(t)$ represent the interference torque and armature current of the right drive motor of the information acquisition robot, and $T_{d l}$ and $i_{l}(t)$ represent the interference torque and armature current of the left drive motor of the information acquisition robot. The angular velocity and control voltage of the right drive motor of the information acquisition robot are represented by $\omega_{r}(t)$ and $u_{r}(t)$, respectively, and the angular velocity and control voltage of the left drive motor of the information acquisition robot are represented by $\omega_{l}(t)$ and $u_{l}(t)$, respectively.

The actuator of the information acquisition robot is the driving motor. The response speed of the driving motor is faster. Ignoring the inductance in the armature circuit of the information acquisition robot, the dynamic equations for the right-wheel drive motor and the left-wheel drive motor of the information acquisition robot are obtained. 


$$
\begin{aligned}
& \frac{R J_{r}(t) \omega_{r}(t)}{R F+k_{t} k_{e}}+\omega_{r}(t)=\frac{k_{t} u_{r}(t)-R T_{d r}(t)}{R F+k_{t} k_{e}} \\
& \frac{R J_{l}(t) \omega_{l}(t)}{R F+k_{t} k_{e}}+\omega_{l}(t)=\frac{k_{t} u_{l}(t)-R T_{d l}(t)}{R F+k_{t} k_{e}}
\end{aligned}
$$

\subsection{Angular Acceleration Control Method}

When the angular acceleration control method for ground-air dual-purpose information acquisition robots is used to control the angular acceleration of the robot, the initial values of the information acquisition robot system and the inverse system are determined based on the dynamic equation. The state equation model of the system is obtained by the $\alpha$-order integral inverse method, the feedback linearization mapping is established, and the non-linear control law is calculated. According to the calculation results, the control model of the angular acceleration regulator of the ground-air dual-purpose information acquisition robot is obtained, and the angular acceleration control of the ground-air dual-purpose information acquisition robot is completed.

Suppose that $\sum$ represents the system of the ground-air dual-purpose information acquisition robot, $u(t)$ represents the input of the information acquisition robot system, and $y(t)$ represents the output of the information acquisition robot system.

$$
\sum:\left\{\begin{array}{l}
x=f(x, u), x\left(t_{0}\right)=x_{0} \\
y=h(x, u)
\end{array}\right.
$$

In Equation (12), $x=f(x, u)$ represents the input function of the information acquisition robot, $y=h(x, u)$ represents the output function of the information acquisition robot, and $x\left(t_{0}\right)=x_{0}$ represents the initial state of the information acquisition robot system. $\hat{\Sigma}$ represents the inverse system of the information acquisition robot system $\sum$. The inverse system $\hat{\Sigma}$ can take the output of the information acquisition robot system as the input of the system and control the input $u_{d}(t)$ of the inverse system, so that the output of the information acquisition robot system is equal to the input $y_{d}(t)$ of the inverse system. Let $\tilde{x}\left(t_{0}\right)=\tilde{x}_{0}$ represent the inverse system of the information acquisition robot. In the initial state of the system, the relationship between the input and output of the inverse system is as follows:

$$
u_{d}(t)=\hat{h}\left(\tilde{x}, y_{d}\right)
$$

$\alpha$-order integral inverse design is the key to achieve angular acceleration control of ground-air dual-purpose information acquisition robots. Equations (14) and (15) are state equation models for ground-air dual-purpose information acquisition robots.

$$
\begin{gathered}
x=f(x)+g(x) u+w(x, u) \\
y=u_{d}(t) c(x)
\end{gathered}
$$

$x(t) \in X \in R^{n}$ represents the state variables of the information acquisition robot system, $X$ represents the correlation set with origin, and represents the state variables set of the information acquisition robot system. $u \in U \in R^{m}$ represents the control variables of the information acquisition robot, $R^{m}$ represents the set of control variables of the information acquisition robot system, $U$ represents the dense set of control variables, $c(x)$ represents the fully differentiable output values, $f(x)$ and $g(x)$ represent the first-order continuous output values, and $w(x, u)$ represents the continuous function.

Let $A(x)$ and $B(x)$ represent decoupling matrices, and the structure matrix $B(x)$ is not uncertain. For any $x$ in the information acquisition robot system, $w(x, u)=0$ is reversible. The feedback linearization mapping of input and output 
of the ground-air dual-purpose information acquisition robot is constructed by decoupling matrices.

$$
v=A(x) / y+B(x) u_{d}(t)
$$

In Equation (16), $v$ represents the pseudo-control variable of the information acquisition robot. Through the pseudo-control variable, the calculation formula for the non-linear control rates $\varphi$ and $\varphi$ of the ground-air dual-purpose information acquisition robot is obtained.

$$
\varphi=-[B(x)]^{-1} A(x)+[B(x)]^{-1} v
$$

The control model $v_{i}$ of the angular acceleration regulator for ground-air dual-purpose information acquisition robots is constructed using Equations (16) and (17). The angular acceleration control of the ground-air dual-purpose information acquisition robot is realized by the control model. The expression of $v_{i}$ is as follows:

$$
v_{i}=\sum_{j=1}^{r_{i}-1} K_{i, j} y_{i}^{r_{i}}+K_{i, j}\left(y_{c i}-\varphi\right)
$$

In Equation (18), $K_{i, j}$ represents the feedback coefficient, and $y_{c i}$ represents the tracking value of the ground-air dual-purpose information acquisition robot system.

\section{Experimental Results and Analysis}

In order to verify the overall effectiveness of the angular acceleration control method for ground-air dual-purpose information acquisition robots, it is necessary to test the angular acceleration control method [14-16]. The operating system tested is Windows, the memory capacity is $8 \mathrm{~GB}$, and the main frequency of CPU is $2.4 \mathrm{GHz}-2.8 \mathrm{GHz}$. In order to accurately control the angular acceleration of ground-air dual-purpose information acquisition robots, the first step is to measure the acceleration [17-18]. The more accurate the acceleration calculation results are, the more accurate the control results are. The angular acceleration control method for the ground-air dual-purpose information acquisition robot (Method 1), the angular acceleration calibration control method for the rotation of the robot (Method 2), and the angular acceleration control method based on trajectory planning (Method 3) are used to test. The acceleration measured by three different methods is compared with the actual acceleration. The test results are shown in Figure 5.

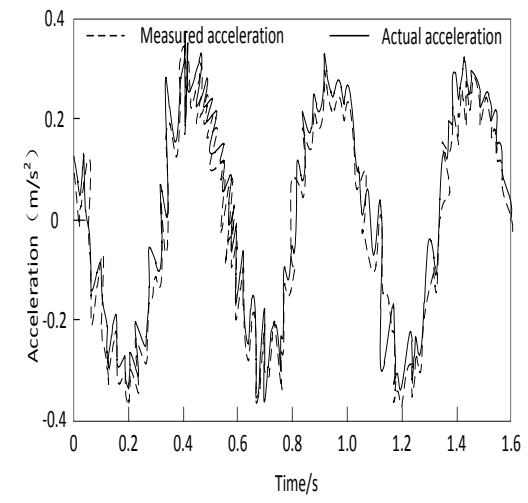

(a) Acceleration measured by Method 1

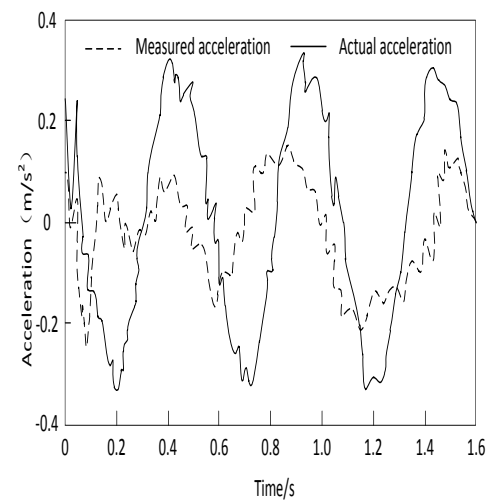

(b) Acceleration measured by Method 2

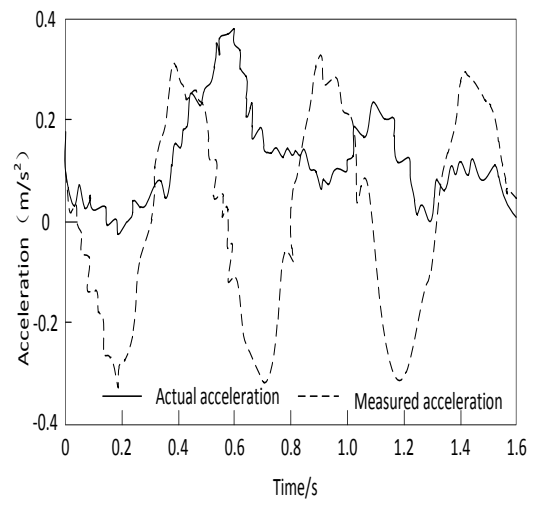

(c) Acceleration measured by Method 3

Figure 5. Acceleration measured by three different methods

Figure 5(a) is the test result of the angular acceleration control method for the ground-air dual-purpose information acquisition robot. Analyzing Figure 5(a), it is known that the angular acceleration control method robot differs little from the actual acceleration. Figures 5(b) and 5(c) are the test results of the angular acceleration calibration control method for the rotation of the robot and the angular acceleration based on trajectory planning, respectively. From the analysis of Figures 5(b) and 5(c), it can be seen that the acceleration measured by the angular acceleration calibration control method for the rotation of the robot and the method of angular acceleration control based on trajectory planning differ greatly from the actual acceleration. Comparing the test results of the three different methods, it can be seen that the angular acceleration control method for ground-air dual-purpose information acquisition robots has higher accuracy, and the control accuracy of 
the angular acceleration control method for ground-air dual-purpose information acquisition robots is higher.

The output voltage is used to test the stability of the control results of the angular velocity control method for the ground-air dual-purpose information acquisition robot (Method 1), the angular acceleration calibration control method (Method 2), and the angular acceleration control method based on trajectory planning (Method 3). The test results are shown in Figure 6.

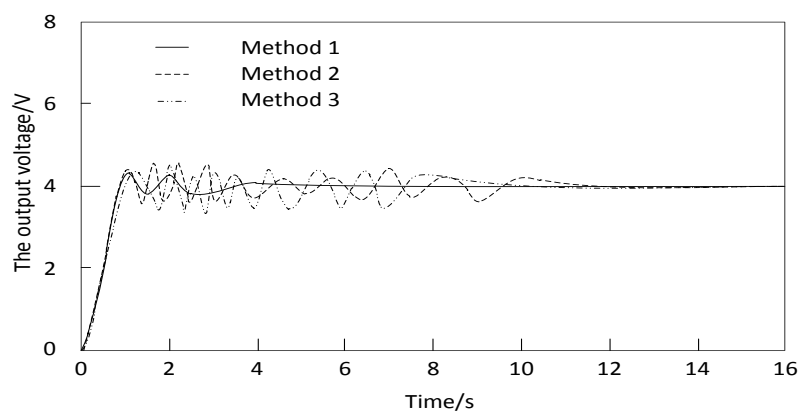

Figure 6. Output voltage of three different methods

Figure 6 shows that when the angular acceleration control method for ground-air dual-purpose information acquisition robots is used to control the angular acceleration of the ground-air dual-purpose information acquisition robot, the output voltage tends to be stable after $4 \mathrm{~s}$, and when the angular acceleration of the ground-air dual-purpose information acquisition robot is controlled by the angular acceleration calibration method for the rotation of the robot, the output voltage tends to be stable after $12 \mathrm{~s}$. When the angular acceleration control method based on trajectory planning is used to control the angular acceleration of the ground-air dual-purpose information acquisition robot, the output voltage tends to be stable after 10s. By comparing the test results of three different methods, it can be seen that the angular acceleration control method of ground-air dual-purpose information acquisition robots can stabilize the output voltage in a relatively short amount of time, which verifies that the angular acceleration control method for ground-air dual-purpose information acquisition robots is more stable.

\section{Conclusions}

Ground-air dual-purpose information acquisition robots are suitable for land and air information acquisition. In order to allow the information acquisition robots to run smoothly, it is necessary to study the angular acceleration control method of the robots. At present, the angular acceleration control method of robots has the problems of low accuracy and stability of control results. A method of angular acceleration control for ground-air dual-purpose information acquisition robots is proposed, and it can accurately measure the acceleration of the information acquisition robot and keep the output voltage stable. It lays a foundation for the development of ground-air dual-purpose information acquisition robots.

\section{Acknowledgments}

This paper is supported by the Tianjin Key R\&D Program Science and Technology Support Key Project (No. S18ZC58678) and Tianjin University Innovation Team Training Program (No. TD13-5094).

\section{References}

1. X. J. Xing, L. L. Huang, D. S. Fan, et al., "Simulation Study on Attitude Optimal Control of Four-Rotor Unmanned Aerial Vehicle," Computer Simulation, Vol. 34, No. 4, pp. 110-114, 2017

2. W. B. Qiu, J. Y. Chai, X. D. Sun, et al., "A Second-Order Differential Angular Acceleration Observer based on Linear Fitting Differential Filter of Position Signal in Series," Journal of Motor and Control, Vol. 20, No. 1, pp. 88-93, 2016

3. S. Liu, Z. Pan, and H. Song, "Digital Image Watermarking Method based on DCT and Fractal Encoding," IET Image Processing, Vol. 11, No. 10, pp. 815-821, 2017

4. Z. Zhang, L. Wang, and C. Zeng, "Attitude Estimation Algorithm of Motion Acceleration Restrained with Dynamic Step Gradient Descent," Information and Control, Vol. 46, No. 2, pp. 136-143, 2017

5. H. Zhao and H. Feng, "Research on a Calibration Method of Rotating Angular Acceleration Sensor," Journal of Sensing Technology, Vol. 29, No. 4, pp. 495-499, 2016

6. J. Peng, Z. Zhang, X. Hu, et al., "Delayed Acceleration Feedback Control of Principal Resonance Response of Piezoelectric Elastic Beams," Vibration and Impact, Vol. 35, No. 24, pp. 1-5, 2016

7. Y. H. Geng and Z. L. H. S. Huang, "Angular Acceleration Trajectory Planning and Vibration Suppression Methods for Flexible 
Satellites," Journal of Astronautics, Vol. 37, No. 12, pp. 1449-1456, 2016

8. S. Liu, "Introduction of Key Problems in Long-Distance Learning and Training," Mobile Networks and Applications, Vol. 24, No. 1, pp. 1-4, 2019

9. Y. Lin, Y. Li, X. Yin, and Z. Dou, "Multisensor Fault Diagnosis Modeling based on the Evidence Theory," IEEE Transactions on Reliability, Vol. 67, No. 2, pp. 513-521, 2018

10. A. I. Diveev, E. Y. Shmalko, and D. N. Zakharov, "Acceleration of the Multilayer Network Operator Method using MPI for Mobile Robot Team Control Synthesis," Procedia Computer Science, Vol. 3, No. 103, pp. 88-93, 2017

11. Z. Pan, S. Liu, and W. Fu, “A Review of Visual Moving Target Tracking,” Multimedia Tools and Applications, Vol. 76, No. 16, pp. 16989-17018, 2017

12. Y. Lin, X. Zhu, Z. Zheng, Z. Dou, and R. L. Zhu, "The Individual Identification Method of Wireless Device based on Dimensionality Reduction and Machine Learning," Journal of Supercomputing, Vol. 75, No. 6, pp. 3010-3027, 2019

13. A. D. Prete and P. Joint, "Velocity Bounds in Discrete-Time Acceleration/Torque Control of Robot Manipulators," IEEE Robotics and Automation Letters, Vol. 3, No. 1, pp. 281-288, 2017

14. Y. Tu, Y. Lin, J. Wang, and J. U. Kim, "Semi-Supervised Learning with Generative Adversarial Networks on Digital Signal Modulation Classification," CMC-Computers Materials and Continua, Vol. 55, No. 2, pp. 243-254, 2018

15. S. Liu, Z. J. Li, and X. C. Cheng, "Introduction of Recent Advanced Hybrid Information Processing," Mobile Networks and Applications, Vol. 23, No. 4, pp. 673-676, 2018

16. S. Kai, G. T. Xu, R. C. Qian, et al., "Error Model of Roll Angle Measurement System for Single Accelerometer High-Spin Projectile," Journal of Detection and Control, Vol. 38, No. 1, pp. 61-65, 2016

17. T. Tsuji, K. Kutsuzawa, and S. Sakaino, "Acceleration Control for Dynamic Manipulation of a Robot Turning Over Objects," IEEE Robotics and Automation Letters, Vol. 4, No. 99, pp. 1, 2017

18. S. Liu, M. Y. Lu, and G. C. Liu, “A Novel Distance Metric: Generalized Relative Entropy,” Entropy, Vol. 19, No. 6, pp. 269, 2017 